\title{
First-principles calculations of the surface relaxation and electronic structure of $\mathrm{Zr}(0001)$
}

\author{
Masahiro Yamamoto \\ Ames Laboratory and Department of Physics and Astronomy, Iowa State University, Ames, Iowa 50011 \\ and Institute of Atomic Energy, Kyoto University, Uji, Kyoto 611, Japan \\ C. T. Chan and K. M. Ho \\ Ames Laboratory and Department of Physics and Astronomy, Iowa State University, Ames, Iowa 50011
}

(Received 10 March 1994)

\begin{abstract}
The multilayer relaxations of the $\operatorname{Zr}(0001)$ surface and the surface electronic structure are studied using first-principles total-energy and force calculations. The distance between the outermost two layers is $4.4-4.7 \%$ smaller than the ideal interlayer spacing, while inner layers show oscillatory relaxations. We examined two possible termination sequences for the surface and found that the hcp surface termination is more stable by about $0.06 \mathrm{eV}$ per surface atom as compared to the fcc terminated structure. We observed an increase in the local density of states (LDOS) for the surface atoms around the Fermi level relative to the bulk atoms. The increase in the LDOS is found to be closely related to the existence of surface states and resonances around the Fermi level.
\end{abstract}

\section{INTRODUCTION}

Knowledge of the geometry and the electronic structure of transition-metal clean surfaces gives a basis to investigate more complex surface phenomena such as oxidation, crystal growth, and catalytic reaction on transition-metal surfaces. However, experimental and theoretical investigations on the clean surfaces of group IV transition metals titanium, zirconium, and hafnium are few. ${ }^{1-4}$ From low-energy-electron-diffraction (LEED) structure analyses, Moore et al. reported that the $\mathrm{Zr}(0001)$ surface has an unreconstructed hexagonal closepacked (hcp) structure and the distance between the outermost two layers is contracted by $(1 \pm 2) \% .^{1}$ Their analysis was limited to the top-layer relaxation, and the results may be affected by possible hydrogen contamination on the surface. ${ }^{5,6}$ Group IV metals have a great affinity for hydrogen, and it is almost impossible to prepare a hydrogen-free clean surface. Hydrogen on the transition-metal surface cannot be detected by the ordinary surface chemical analysis such as Auger electron spectroscopy but recently Garrett et al. have shown the existence of residual hydrogen adatom on a surface-chemical-analytically clean $\mathrm{Ti}(0001)$ surface by using high resolution electron energy loss spectroscopy. ${ }^{5}$

On the theoretical side, using the embedded atom method (EAM) Chen recently found that the outermost layer distance of the $\operatorname{Zr}(0001)$ surface expanded by $1.84 \% .^{7}$ Methfessel et al. reported the top-layer surface relaxation, work function, and surface energy for the $4 d$ transition-metal series from first principles linear muffin-tin orbital (LMTO) calculations. ${ }^{3}$ However, their calculations were carried out for the face-centered cubic (fcc) $\operatorname{Zr}(111),(110)$, (100) surfaces. The outermost twolayer distance for the fcc (111) surface is contracted by $2.5 \%$. For the structure of hcp $\operatorname{Zr}(0001)$ which has dif-

ferent stacking sequences and a different $c / a$ ratio from the fcc structure, it seems that there are no conclusive results at present. One purpose of this work is to determine the fully relaxed surface structure with a first-principles total-energy and force method which has been shown to give an accurate description of the structures of solid and solid surfaces. ${ }^{8,9}$

The electronic structure of the zirconium surface has not been studied in detail experimentally and theoretically. Feibelman et al. have calculated the electronic structure of the unrelaxed $\mathrm{Ti}(0001)$ surface ${ }^{2}$ and recently Lee et al. calculated the electronic structure of the unrelaxed $\operatorname{Zr}(0001)$ surface. ${ }^{4}$ Both studies found a large increase in the surface local density of states (LDOS) around the Fermi level. In the present study, the same behavior of the LDOS for the fully relaxed $\operatorname{Zr}(0001)$ surface is also observed. We specify the surface states and resonance along the high symmetry $\bar{\Gamma}-\bar{M}-\bar{K}$ lines, together with the projected bulk band structure in the twodimensional Brillouin zone.

\section{FIRST-PRINCIPLES TOTAL-ENERGY AND FORCE CALCULATIONS}

The first-principles calculations have been carried out using a pseudopotential method within the framework of the density functional theory. ${ }^{10,11}$ The Hedin-Lundqvist form of exchange-correlation functionals has been used. The Bloch wave functions are expanded in a mixed basis of plane waves and numerical localized wave functions centered on atomic sites. ${ }^{12}$ We used a plane wave basis with kinetic energy up to $15 \mathrm{Ry}$. Details of the total energy calculation are given in Ref. 12 .

The zirconium surface system is represented by a supercell with repeated slabs of eight and ten $\operatorname{Zr}(0001)$ lay- 
ers, separated by a vacuum region with thickness corresponding to five layers. We found the charge density is negligibly small in the middle of the vacuum region. We used six special $\mathbf{k}$ points in the two-dimensional irreducible Brillouin zone for $\mathbf{k}$ points summation. ${ }^{13}$ Our calculation results show that there are no significant differences in the total-energy surface-layer relaxation curves in the 6,18 special $\mathbf{k}$ points, and 20 evenly sampled $\mathbf{k}$ points: the surface energies differ by about $35 \mathrm{meV}$ and the top layer relaxations differ by less than $1.5 \%$ of the interlayer spacing.

All the interlayer distances are fully relaxed using forces calculated via the Hellmann-Feynman theorem. ${ }^{14}$ The convergence of the force with respect to selfconsistency is accelerated with a recently proposed scheme.$^{15}$ Relaxations of atomic positions are performed with a Broyden's scheme where the initial force matrix is determined from bulk force constants and surface force constants from preliminary calculations. Initial input potentials are predicted as prescribed by Ref. 15 .

\section{RESULTS AND DISCUSSION}

We first considered the energetics of bulk zirconium. In Fig. 1 the dependence of the total energies on the atomic volume are shown for the hexagonal close-packed (hcp), hexagonal $\omega$-phase, face-centered-cubic (fcc), and body-centered-cubic (bcc) structures of zirconium. The calculations were carried out with 80 (hcp and $\omega$ ), 60 (fcc), and 70 (bcc) evenly sampled $\mathbf{k}$ points in the irreducible Brillouin zone. In the hcp and $\omega$ phases, the

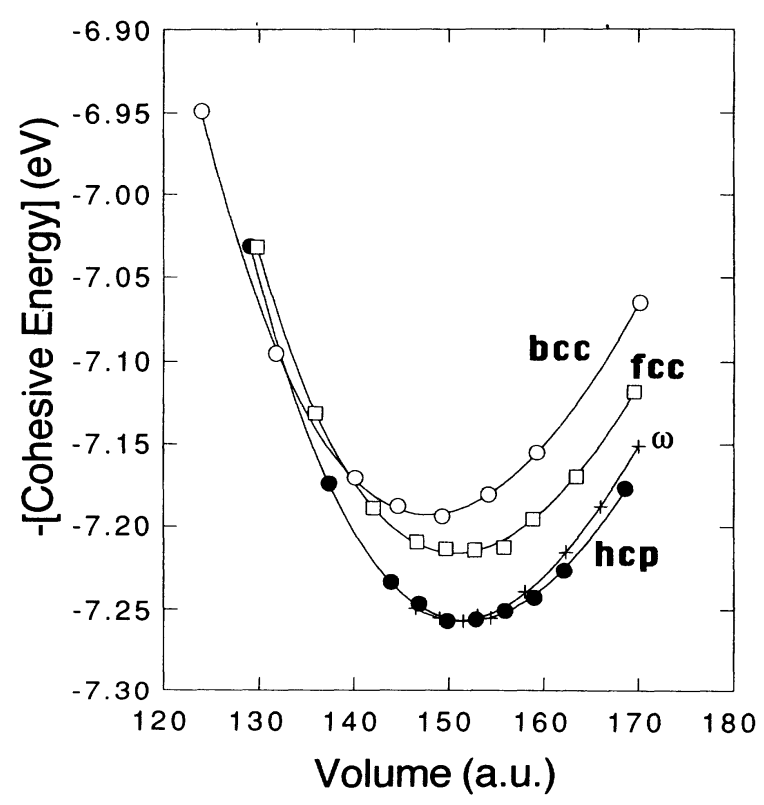

FIG. 1. Volume dependence of the cohesive energy of bulk zirconium. The $c / a$ ratios of hcp and $\omega$ zirconium are 1.61 and 0.62 , respectively. The volume and the cohesive energy shown are those for one atom. $c / a$ (long axis to short axis) ratios are determined to be 1.61 and 0.62 , respectively, by parabolic fits to the curves of total energy at the volume which minimizes the total energy. By fitting a "universal binding curve" to our calculated energies, we determine the optimized lattice constants, cohesive energies, and bulk moduli. ${ }^{12}$ The results are shown in Table I. The calculation results are in good agreement with experiment.

The cohesive energies of the hcp phase and the $\omega$-phase zirconium differ by less than $0.5 \mathrm{meV}$ per atom. The two structures have nearly the same energy within the accuracy of our calculation. ${ }^{18}$ The physical parameters calculated for bulk zirconium are as follows. The Poisson ratio $-e_{x x} / e_{z z}\left[=-C_{13} /\left(C_{11}+C_{12}\right)\right]$ subject to a $c$-axis stress $Z_{z}$ is estimated to be 0.34 [experiment 0.29 (Ref. 21)] for hcp zirconium. The ratio was estimated by determining the $a$-axis length with a minimum total energy for a given $c$-axis length. ${ }^{25} \mathrm{We}$ also estimated the ratio $-e_{z z} / e_{x x}\left(=-2 C_{13} / C_{33}\right)$ subject to a uniform stress $X_{x}=Y_{y}=P_{\|}$in the $x-y$ plane is 0.83 [experiment 0.75 (Ref. 21)] for hcp zirconium. This ratio was estimated by determining the $c$-axis length with a minimum total energy for a given $a$-axis length. The density of states (DOS) at the Fermi level for hcp, $\omega$, fcc, and bcc zirconium are 11.6, 13.3, 21.3, and 20.9 states/atom/Ry, respectively. The DOS for hcp, $\omega$, fcc, and bcc zirconium were calculated with the linear tetrahedron interpolation scheme $^{26}$ by using $936,936,916$, and $910 \mathrm{k}$ points in the irreducible Brillouin zone, respectively. The phonon enhancement parameter $(1+\lambda)$, which may be defined by $^{27,28}$

$$
(1+\lambda)=3 C_{\text {el }}(\text { measured }) /\left[\pi^{2} k_{B}^{2} T D\left(E_{F}\right)\right]
$$

is estimated to be 1.38 for hcp zirconium. Here, $C_{\mathrm{el}}$ is the specific heat of electron and $D\left(E_{F}\right)$ is the DOS at the Fermi level. The enhancement parameter is in agreement with McMillan's estimation 1.41, which was determined from the superconducting properties of zirconium. ${ }^{27}$

The ideal slab structure is constructed with the lattice constants calculated above. The deviation of $\Delta a / a, \Delta c / c$, and $\Delta(c / a) /(c / a)$ from the experimental results are $-1.4 \%,-0.3 \%$, and $+1.1 \%$, respectively. ${ }^{19}$ We usually use calculated lattice constants rather than experimental lattice constants in surface calculations to avoid having artificial macroscopic stress in our slabs. Otherwise part of the relaxation actually reflects the difference between local density approximation (LDA) and experimental lattice constants, rather than the effects of creating a surface.

The slab structures of two different stacking sequences, i.e., hcp unreconstructed $(A B A B A B A B)$ and fcc reconstructed $(C B A B A B A C)$ are considered here. Eight (hcp and fcc reconstructed sequences) and ten (hcp sequence only) layers slabs with inversion symmetry are used. Starting from the ideal structure and keeping the $(1 \times 1)$ symmetry, the interlayer distances are fully relaxed. The surface relaxation, the surface energy and the work function calculated are shown in Table II. The relaxation $\Delta_{i j}$ is defined by the change of the interlayer distance between the $i$ th and $j$ th layer. The surface en- 
ergy is defined by $\left(E_{\text {tot }}-n_{\text {atom }} E_{\mathrm{Zr}}\right) / 2$, where $E_{\text {tot }}$ is the total energy of the slab, $n_{\text {atom }}$ is the number of zirconium atoms in the slab, and $E_{\mathbf{Z r}}$ is the total energy per atom of hcp zirconium in equilibrium. The factor of 2 is because a slab is bounded by two surfaces. The work function is determined by the energy difference between the Fermi energy and the potential energy at the middle point in the vacuum region. Although the hcp structure is more stable than the fcc structure in bulk zirconium, it is conceivable that in the rather drastic process of surface formation, the top layer may choose to have a fcc termination (i.e., $A B C$ ) relative to the inner layers rather than a hcp termination $(A B)$. Thus, we considered this possible reconstruction where the surface unit cells remains $(1 \times 1)$. The hcp surface structure of the slab with eight layers $(A B A B A B A B)$ is found to be more stable by 60 meV per surface atom than the fcc reconstructed surface structure $(C B A B A B A C)$. This is in agreement with the LEED structure analysis. ${ }^{1}$ This energy difference is also consistent with the stacking fault energy in the slab. If we assume that the nearest-neighbor interlayer interaction determines the energy of the close-packed stacking sequence system, ${ }^{31}$ the energy difference between the hcp and fcc-reconstructed surface structure may be described by an axial-nearest-neighbor Ising model, ${ }^{32,33}$

$$
E=\sum_{i} J_{i} s_{i} s_{i+1}
$$

The sequences $A \triangleright B \triangleright C \triangleright A \triangleright$ and $A \triangleright C \triangleright B \triangleright A \triangleright$ are described by Ising spin 1 and -1 , respectively. ${ }^{33}$ The energy difference between the hcp (0001) structure and the fcc reconstructed structure becomes $2 J$ per surface atom, if the interaction $J_{i}$ between the nearest-neighbor Ising spins is assumed to be the same $J$. The interaction $2 J$, which is equivalent to the energy difference $40 \mathrm{meV}$ per atom between the bulk hcp and the bulk fcc structure in this model, is close to the energy difference of the two slabs.

The assumption of nearest-neighbor interlayer interaction may be justified by the charge density distribution of the slabs. In Fig. 2 and Fig. 3 the (pseudo)charge distribution and the difference between the charge of the slab and that of a superposition of pseudoatomic charge are shown for the two different slab structures, respectively. The charge difference in the third and fourth layer region is almost the same as the hcp bulk structure. The charge and its difference map outside the surface (electron smearing into the vacuum region) are also the same except for the atomic position difference of the outermost layer. The only major difference between the two slabs

TABLE I. Comparison of calculated physical parameters of bulk zirconium with experimental results and other calculated results.

\begin{tabular}{|c|c|c|c|c|c|}
\hline & \multicolumn{2}{|c|}{ Lattice constant } & \multirow[b]{2}{*}{$c / a$} & \multirow{2}{*}{$\begin{array}{c}\text { Cohesive energy } \\
(\mathrm{eV})\end{array}$} & \multirow{2}{*}{$\begin{array}{l}\text { Bulk modulus } \\
\qquad(\mathrm{GPa})\end{array}$} \\
\hline & $a$ (a.u.) & $c$ (a.u.) & & & \\
\hline \multicolumn{6}{|l|}{ hcp Zr } \\
\hline present cal. & 6.017 & 9.688 & 1.61 & 7.26 & 110 \\
\hline cal. $^{\mathbf{a}}$ & 6.05 & 9.69 & 1.60 & 6.98 & 101 \\
\hline cal. $^{\mathrm{b}} H-L$ & 5.943 & 9.668 & 1.627 & 7.40 & 98.6 \\
\hline$X \alpha$ & 6.111 & 9.872 & 1.608 & 6.04 & 84.6 \\
\hline cal. $^{\mathrm{c}}$ & 5.995 & 9.627 & 1.606 & & \\
\hline experimental & $6.1027^{d}$ & $9.7159^{d}$ & $1.592^{\mathrm{d}}$ & $6.25^{\mathrm{e}}$ & $97.2^{\mathrm{f}}$ \\
\hline \multicolumn{6}{|l|}{ fcc $\mathrm{Zr}$} \\
\hline present cal. & 8.448 & & & 7.22 & 108 \\
\hline cal. ${ }^{\mathrm{g}}$ & 8.32 & & & 6.76 & 94 \\
\hline \multicolumn{6}{|l|}{ bcc Zr } \\
\hline present cal. & 6.662 & & & 7.19 & 104 \\
\hline cal. $^{a}$ & 6.69 & & & 6.93 & 105 \\
\hline experimental $^{\mathrm{h}}$ & 6.755 & & & & 97 \\
\hline \multicolumn{6}{|l|}{$\omega \mathrm{Zr}$} \\
\hline present cal. & 9.447 & 5.857 & 0.62 & 7.26 & 116 \\
\hline cal. $^{c}$ & & & 0.617 & & \\
\hline experimental $^{\mathrm{i}}$ & 9.52 & 5.9 & 0.62 & & 110 \\
\hline
\end{tabular}

${ }^{\mathrm{a}}$ Reference 16.

${ }^{\mathrm{b}}$ Reference 17 .

${ }^{\mathrm{c}}$ Reference 18.

${ }^{\mathrm{d}}$ Reference 19.

${ }^{\text {e }}$ Reference 20.

${ }^{\mathrm{f}}$ Reference 21 .

${ }^{g}$ Reference 22 .

${ }^{\mathrm{h}}$ Reference 23. The lattice constant is the extrapolated value at $0 \mathrm{~K}$. The bulk modulus is the value at $1188 \mathrm{~K}$.

${ }^{\mathrm{i}}$ Reference 24 . 


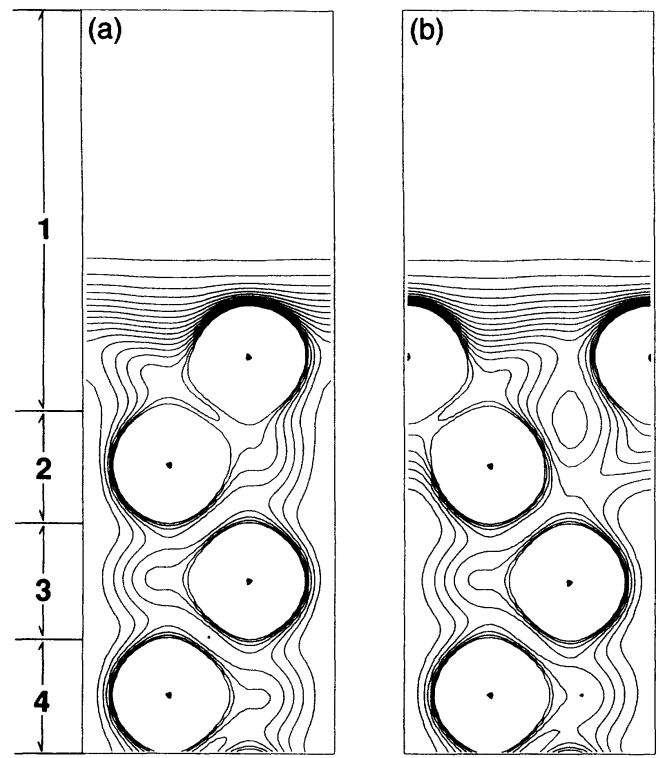

FIG. 2. Charge density contour plot for: (a) hcp zirconium slab; (b) fcc reconstructed slab. The plane is defined by the vectors [1-100] and (1/2)[0001]. Charge densities on successive contours differ bv 0.002 electrons $/(\text { a.u. })^{3}$. is the charge difference distribution in the interstitial region between the first-second and the second-third layers. This fact may support the nearest-neighbor interlayer interaction assumption and that the energy difference between the two slab structures can be explained by the stacking fault energy.

The surface energy calculated for hcp surface is $\mathbf{1 . 1 2}$ $\mathrm{eV}$ per atom for the slabs with eight as well as ten layers. This value is higher than the surface tension of the liquid zirconium surface (0.81 eV per atom) (Ref. 29) and lower than the value (1.56 eV per atom) estimated by the equation $N_{s} E_{\mathrm{Zr}}\left(Z-Z_{s}\right) / Z$ (simple bond-cutting model). Here, $E_{\mathrm{Zr}}$ is the experimental cohesive energy, $Z$ is the coordination number of zirconium in the bulk, $Z_{s}$ is the coordination number of the surface atom, and $N_{s}$ is the atomic density of the surface. It is known that the simple bond-cutting model overestimates the surface energy, ${ }^{3}$ because charge redistribution effects caused by the creation of the surface are not taken into account. This is clearly shown by the charge redistribution map in Fig. 3. The surface tension of liquid metal is usually smaller than the surface energy of the solid at $0 \mathrm{~K}$, because of entropy effects. The surface tension is given $\mathrm{by}^{34}$

$$
\gamma(0)=\gamma\left(T_{m}\right)+\left.\int_{0}^{T_{m}}(S / A) d T\right|_{\epsilon=\text { const }}
$$

TABLE II. Comparison of calculated zirconium surface physical parameters with experimental results and other calculations. Daggers represent the change in the spacing between the innermost two layers of the slabs with eight and ten layers.

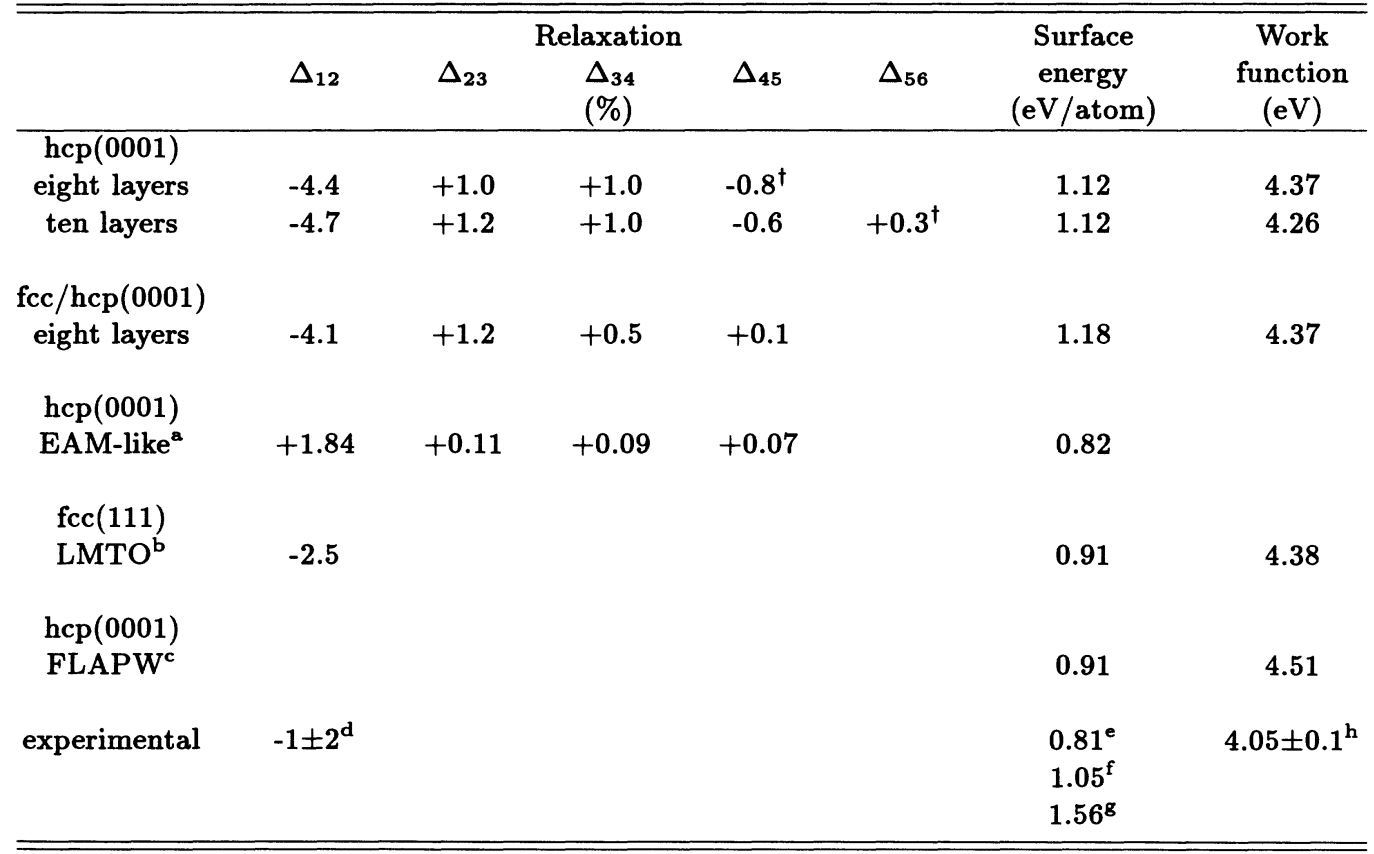

${ }^{\mathrm{a}}$ Reference 7 .

${ }^{\mathrm{b}}$ Reference 3 .

${ }^{\mathrm{c}}$ All-electron full-potential linearized augmented (FLAPW) method, Ref. 4.

${ }^{\mathrm{d}}$ Reference 1.

${ }^{\text {e}}$ Liquid zirconium surface tension, Ref. 29.

${ }^{f}$ Extrapolated value to $0 \mathrm{~K}$ from the liquid metal surface tension (Ref. 29).

${ }^{\mathrm{g}}$ Estimated by $E_{\mathrm{Zr}}\left(Z_{s} / Z\right) N_{s}$, see text.

${ }^{\text {h}}$ Polycrystalline Zr surface, Ref. 30. 

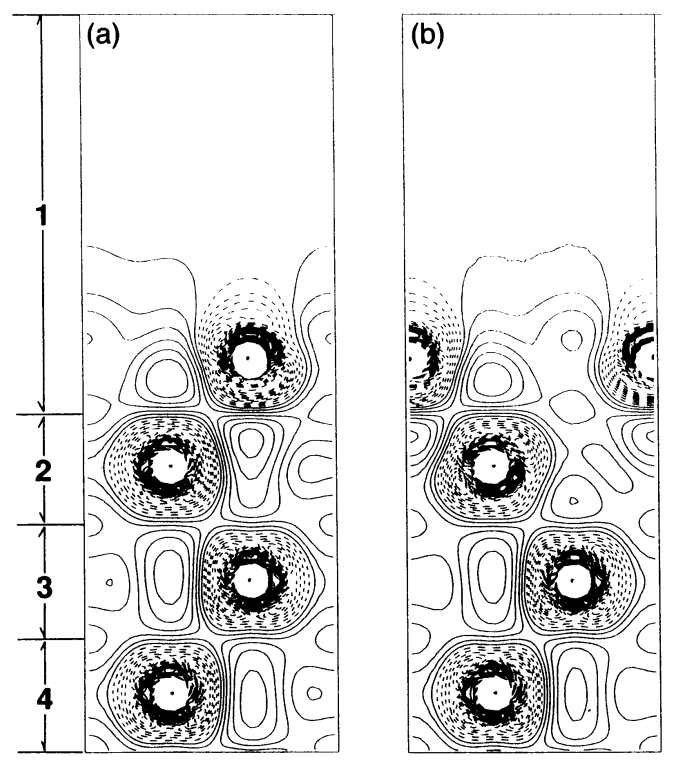

FIG. 3. Charge distribution difference between the slab and the superposition of pseudoatomic charge density for: (a) hcp slab; and (b) fcc reconstructed slab. Solid and dashed contours correspond to the regions of charge accumulation and depletion, respectively. The plane spanned by the vectors $[1-100]$ and $(1 / 2)[0001]$ is shown. Charge densities on successive contours differ by 0.002 electrons $/($ a.u. $) .^{3}$

Here, $\gamma$ is the surface tension, $T_{m}$ is the melting point of the metal, $S$ is the entropy of the surface system, $A$ is the surface area, and $\epsilon$ is the surface strain. Since the argument of the integral is positive, the surface tension (surface energy) of metal surface at $0 \mathrm{~K}$ is higher than the value of the liquid surface. If the linear dependence of the surface tension of liquid zirconium on temperature would hold in the solid zirconium temperature region, the surface tension at $0 \mathrm{~K}$ is estimated to be $1.05 \mathrm{eV}$ per atom. ${ }^{29}$ This value is in better agreement with our calculated value.

Like most other transition-metal clean surfaces, the relaxation of the outermost two layer spacing $\Delta_{12}$ for the hcp zirconium surface shows a $4.4 \%$ (4.7\% for the ten layers slab) contraction in our calculation. The contraction is greater than the experimental value of $(-1 \pm 2) \%$. However, considering the uncertainty of the LEED structure analysis ${ }^{34}$ and the possible hydrogen adsorption effects, the agreement between the measured and the calculated relaxation values is acceptable. The fcc surfaces of $\mathrm{Zr}$ have been studied by a recent LMTO calculation, and for the (111) (110) (100) surfaces the outermost two layer spacings also show contraction, and the $\Delta_{12}$ are $-2.5,-4.2$, and $-5.5 \%$, respectively. ${ }^{3}$

There are several simple models to explain the inward surface relaxation of transition metals: (i) Smoluchowski smoothing, ${ }^{35,36}$ which reduces the curvature of the charge density of the surface and decreases the electron kinetic energy, can be observed in Figs. 2 and 3. Lee et al. also reported the smoothness of the calculated charge density contour of the $\operatorname{Zr}(0001)$ surface. $^{4}$
The charge density distribution in the outermost charge contour is made more flat as compared to the superposition of pseudoatomic charge densities (not shown), and the smoothing arises from the charge transfer from the region just above the surface atoms to the hollows between the surface atoms as shown in Fig. $3 .^{36,37}$ For a layer geometry like the hcp (0001) surface, this would lead to an oscillatory relaxation of the inner layers. ${ }^{36,38}$ (ii) In addition, the electrons involved in the surface bond-cutting backbond to the subsurface layer atoms. The backbonding reduces the interlayer distance between the first and second layer. ${ }^{37,39}$ As shown in Fig. 3 the charge density flow to the interstitial between the first and second layer is greater than that in the innermost bulklike layer region.

By using a method similar to the EAM model Chen calculated the multilayer relaxation of hcp (0001) metal surface. ${ }^{7}$ His calculation for the relaxations $\Delta_{12}, \Delta_{23}, \Delta_{34}$, and $\Delta_{45}$ of zirconium hcp (0001) surface shows all expansions: $1.84,0.11,0.09$, and $0.07 \%$, respectively. In general, EAM-like models are empirical and depend on the transferability of the fitted parameters, and thus are less reliable as compared with local density calculation results. ${ }^{40}$

The inner-layer relaxations $\Delta_{23}, \Delta_{34}$ are found to be small $(1.0 \%, 1.0 \%$ for eight layers and $1.2 \%, 1.0 \%$ for ten layers slab, respectively). The oscillatory relaxations are similar to other metal surfaces. ${ }^{34,39}$ The energy difference of the ideal and the fully relaxed structure is $30 \mathrm{meV}$ for the hcp structure, and the energy gain is small when compared to the surface energy (the ratio is $2.7 \%$ ). From the interplanar force constants ${ }^{41}$ we have found the interlayer couplings in this slab are decreased by a factor of ca. 10 with each successive neighboring layer. The vibrational properties of the slab will be reported elsewhere.

The work function is calculated to be $4.4 \mathrm{eV}(4.3 \mathrm{eV}$ for the hcp slab with ten layers) for both the hcp and fcc-reconstructed zirconium surfaces. The experimental value for polycrystalline zirconium surface is $4.05 \pm 0.1$ $\mathrm{eV},{ }^{30}$ which again may be affected by hydrogen adsorption. In the case of $\operatorname{Ti}(0001)-(1 \times 1) \mathrm{H}$, the change in the work function is predicted to be $\pm 0.4 \mathrm{eV}$, and the change is strongly dependent on the location of the adsorption site. $^{42}$

The layer-decomposed local density-of-states are evaluated using the following definition:

$$
D(i, E) \equiv \sum_{\mathbf{k}_{\|}, n} \int_{\Omega_{i}} d \mathbf{r} \rho_{n}\left(\mathbf{k}_{\|}, \mathbf{r}\right) \delta\left(E-E_{n}\left(\mathbf{k}_{\|}\right)\right) .
$$

Here, $\mathbf{k}_{\|}$is a wave vector in the two-dimensional Brillouin zone, $n$ is a band index, $\Omega_{i}$ is the region corresponding to the $i$ th layer, and $\rho_{n}\left(\mathbf{k}_{\|}, \mathbf{r}\right)$ is the charge density of the state $E_{n}\left(\mathbf{k}_{\|}\right) . \rho_{n}\left(\mathbf{k}_{\|}, \mathbf{r}\right)$ is normalized to one in the unit cell. The summation of $k_{\|}$was done over 66 evenly spaced $\mathbf{k}$ points in the two-dimensional Brillouin zone, and 80 bands were considered. The $\delta$ function in the Eq. (3) is replaced by a Gaussian smearing function with 0.15 $\mathrm{eV}$ width.

The calculated LDOS for the hcp slab with eight layers is shown in Fig. 4. The regions 1-4 correspond to the 


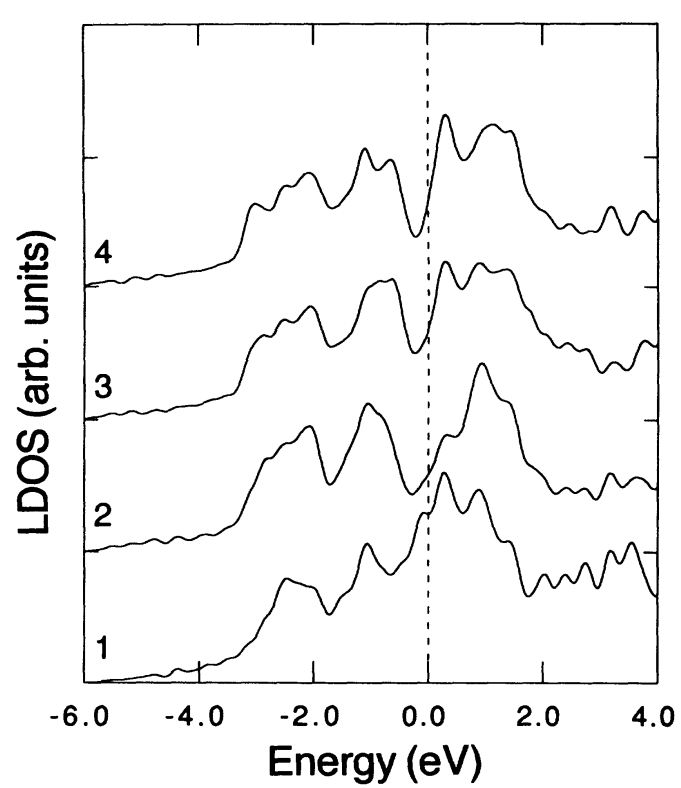

FIG. 4. Local density of states (LDOS) of zirconium hcp slab. The energy zero corresponds to the Fermi level.

regions shown in Fig. 2. Although the density of states of bulk hcp zirconium has a minimum near the Fermi energy ( $0.2 \mathrm{eV}$ below $E_{F}$ in our hcp calculation), the LDOS around the Fermi level is increased at the surface layer. The LDOS of the third and fourth layer is almost the same as the bulk DOS. The increase in the LDOS around the Fermi level at the surface layer is accompanied by a small decrease of the second layer LDOS around the Fermi level. The narrowing of the band due to the reduced coordination at the surface layer can also be seen in the energy range from $-3 \mathrm{eV}$ to $2 \mathrm{eV}$ in the LDOS. Tapping reported angle-integrated ultraviolet photoemission spectra for a clean $\mathrm{Zr}(0001) .{ }^{43}$ There are three peaks in this spectra, i.e., 1, 3, and $6 \mathrm{eV}$ below the Fermi energy. The two peaks of the spectra with energies 1 and 3 $\mathrm{eV}$ below the Fermi energy are in good agreement with our LDOS results. The peak at $6 \mathrm{eV}$ below the Fermi level may be caused by hydrogen contamination at the surface, because there is no peak in the calculated DOS around the corresponding energy for the surface and bulk zirconium. The increase of the LDOS around the Fermi level in the top layer indicates there are surface states and resonances near the Fermi level, as will be shown along some high symmetry directions.

In Fig. 5 the surface states (closed circles) and resonances (open circles) along the high symmetry line of the surface Brillouin zone of the fully relaxed zirconium surface are shown. The shaded regions in Fig. 5 show the projected band structure of the bulk hcp zirconium. The surface states and the resonances are determined by the criterion that the population of the wave function in the region 1 (surface layer) exceeds $50 \%$. The dispersionless surface states and resonances around the Fermi energy increase the LDOS of the surface layer. The surface states and resonances calculated here are similar to

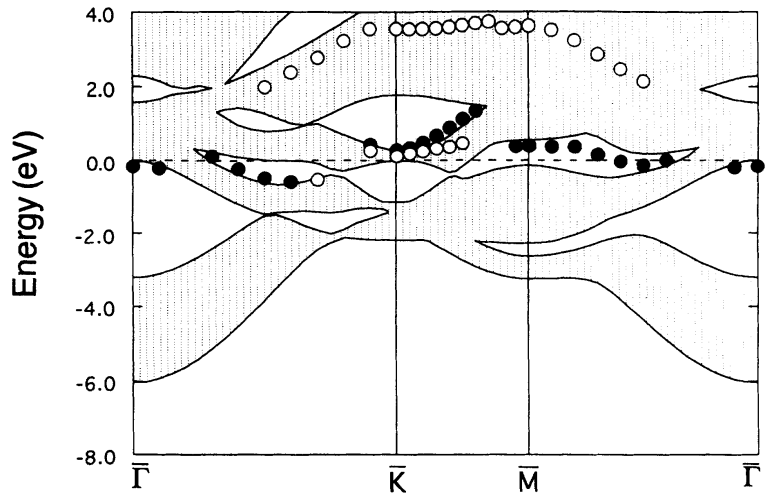

FIG. 5. Band structure of zirconium hcp slab along the high symmetry line in the surface Brillouin zone. Open and closed circles correspond to the surface resonances and the surface states in the gap of the projected band structure of bulk hcp zirconium, respectively. The shaded areas indicate the region where bulk states can exist. The energy zero corresponds to the Fermi level.

those of the $\mathrm{Ti}(0001)$ surface calculated by Feibelman et al.: (i) A small portion of the surface Brillouin zone around the $\bar{\Gamma}$ point has a surface state close to the Fermi level; and (ii) the surface state near the middle of the $\bar{\Gamma}-\bar{K}$ line and the surface state starting from the $\bar{M}$ point to the middle of $\bar{M}-\bar{\Gamma}$ line correspond to the same band, and merges into the bulk states continuum around the $\bar{K}$ point. This surface band has a maximum energy around the $\bar{M}$ point and is unoccupied. This band is flat and contributes to the LDOS of the surface layer around the Fermi level. However, there are also some differences: (1) The energy level of the surface states and the resonances around the $\bar{K}$ point to the $\bar{K}-\bar{M}$ line are located above the Fermi level and (2) the existence of the resonances with energy dispersion 2-4 eV above the Fermi level (this is also reflected in the increase of the top layer LDOS in the range $2-4 \mathrm{eV}$ above the Fermi level).

In Fig. 6, the charge density distribution of the sur-

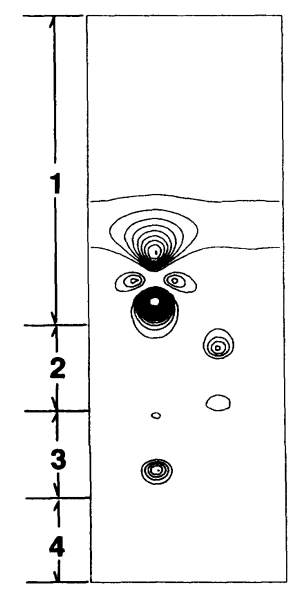

FIG. 6. Charge contour plot of the surface state at the $\bar{\Gamma}$ point. The plane spanned by the vectors $[1-100]$ and $(1 / 2)[0001]$ is shown. 
face states at the $\bar{\Gamma}$ point is shown. From the charge density contour, this state is mostly of $d_{3 z^{2}-r^{2}}$ character. No angle-resolved ultraviolet photoemission results for $\operatorname{Zr}(0001)$ surface have been reported to our knowledge. For the titanium (0001) surface, the normal emission of photoelectrons, where the initial state corresponds to the $\bar{\Gamma}$ point states, has a strong peak very close to the Fermi level and no dispersion with the incident photon energy. ${ }^{44}$ This state shows a strong emission for incident UV light with $p$ polarization, but the emission is reduced for incident light with $s$ polarization. ${ }^{44}$ Hermanson showed that for the photoemission normal to the surface the symmetry of the initial electron state ( $\bar{\Gamma}$ point state $)$ is the same as that of the dipole operator causing the optical transition, ${ }^{45}$ i.e., $s-, p_{z^{-}}$, and $d_{3 z^{2}-r^{2}}$-like initial states can be emitted by light with $p$ polarization, and $p_{x}, p_{y}$ and $d_{x^{2}-y^{2}}$-like states by $s$-polarized light. The $\bar{\Gamma}$ point surface state with $d_{3 z^{2}-r^{2}}$ character found in the present study is consistent with the results of the $\operatorname{Ti}(0001)$ surface. A similar surface state was also observed on the Co(0001) surface. ${ }^{46}$ Since this surface state exists in a small portion of the Brillouin zone around the $\bar{\Gamma}$ point, it does not have a large contribution to the LDOS.

In summary, we used the first-principles calculations to determine the fully relaxed structure of the clean zirconium (0001) surface. The calculation showed that the unreconstructed hcp structure with more than $4 \%$ surface layer contraction is the energetically most favorable structure. The top-layer relaxation, the surface energy, the work function, and the surface electronic structure are in agreement with experiments. We found that the surface states and the resonances increase the surface LDOS around the Fermi energy.

\section{ACKNOWLEDGMENTS}

One of us (M.Y.) would like to thank Ames Laboratory for their hospitality during his visit for one year. Ames Laboratory is operated for the U.S. Department of Energy by Iowa State University under Contract No. W-7405-ENG-82. This work is supported by the Director of Energy Research, Office of Basic Energy Sciences, and Advanced Energy Projects including a grant of computer time on the Cray computers at NERSC at the Lawrence Livermore Laboratory. Computations were performed in part at Supercomputer Facility, Institute of Atomic Energy, Kyoto University.
${ }^{1}$ W. T. Moore, P. R. Watson, D. C. Frost, and K. A. R. Mitchell, J. Phys. C 12, L887 (1979).

${ }^{2}$ P. J. Feibelman, J. A. Appelbaum, and D. R. Hamann, Phys. Rev. B 20, 1433 (1979).

${ }^{3}$ M. Methfessel, D. Hennig, and M. Scheffler, Phys. Rev. B 46, 4816 (1992).

${ }^{4}$ J. I. Lee, S. K. Hwang, S. C. Hong, and A. J. Freeman, in Physics of Transition Metals (ICPTM92), edited by P. M. Oppenneer and J. Kübler (World Scientific, Singapore, 1993), pp. 520-523.

${ }^{5}$ S. J. Garrett, R. G. Egdell, and J. C. Rivière, J. Electron Spectrosc. Relat. Phenom. 54/55, 1065 (1990).

${ }^{6}$ P. J. Feibelman and D. R. Hamann, Surf. Sci. 234, 377 (1990).

${ }^{7}$ S. P. Chen, Surf. Sci. 264, L162 (1992).

${ }^{8}$ G. P. Srivastava and D. Weaire, Adv. Phys. 36, 463 (1987).

${ }^{9}$ K. M. Ho and B. N. Harmon, Mater. Sci. Eng. A 127, 155 (1990); Noboru Takeuchi, C. T. Chan, and K. M. Ho, Phys. Rev. B 43, 13899 (1991); Y. G. Ding, C. T. Chan, and K. M. Ho, Phys. Rev. Lett. 67, 1454 (1991); C. T. Chan, K. P. Bohnen, and K. M. Ho, ibid. 69, 1672 (1992).

${ }^{10}$ See, e.g., R. O. Jones and O. Gunnarsson, Rev. Mod. Phys. 61, 689 (1991).

${ }^{11}$ D. R. Hamman, M. Schlüter, and C. Chiang, Phys. Rev. Lett. 43, 1494 (1979); G. P. Kerker, J. Phys. C 13, L189 (1980). The norm-conserving pseudopotential of zirconium are generated by regarding $4 p$ states as valence states in order to reduce the size of core region $[Y$. Y. Ye (private communication)]. The bulk structure and relaxation of surface structure have no significant differences compared with those calculated generated by treating the $4 p$ as core states.

${ }^{12}$ C. Elsässer, N. Takeuchi, K. M. Ho, C. T. Chan, P. Braun, and M. Fähnle, J. Phys. Condens. Matter 2, 4371 (1990).

${ }^{13}$ S. L. Cunningham, Phys. Rev. B 10, 4988 (1974).

${ }^{14}$ K. M. Ho, C. Elsässer, C. T. Chan, and M. Fähnle, J. Phys. Condens. Matter 4, 5189 (1992).
${ }^{15}$ C. T. Chan, K. P. Bohnen, and K. M. Ho, Phys. Rev. B 47, 4771 (1993)

${ }^{16}$ Y. Chen, C. L. Fu, K. M. Ho, and B. N. Harmon, Phys. Rev. B 31, 6775 (1985).

${ }^{17}$ Z. W. Lu, D. Singh, and H. Krakuaer, Phys. Rev. B 36, 7335 (1987).

${ }^{18}$ R. Ahuja, J. M. Wills, B. Johansson, and O. Eriksson, Phys. Rev. B 48, 16269 (1993).

19 J. Goldak, L. T. Lloyd, and C. S. Barrett, Phys. Rev. 144, 478 (1966).

${ }^{20}$ C. Kittel, Introduction to Solid State Physics, 6th ed. (Wiley, New York, 1986).

${ }^{21}$ E. S. Fisher and C. J. Renken, Phys. Rev. 135, A482 (1964).

${ }^{22}$ V. L. Moruzzi, J. F. Janak, and A. R. Williams, Calculated Electronic Properties of Metals (Pergamon, New York, 1978).

${ }^{23}$ A. Heiming, W. Petry, J. Trampenau, M. Alba, C. Herzig, H. R. Schober, and G. Vogl, Phys. Rev. B 43, 10948 (1991).

${ }^{24}$ S. K. Sikka, Y. K. Vohra, and R. Chidambaram, Prog. Mater. Sci. 27, 245 (1982).

${ }^{25}$ J. R. Chelikowsky, C. T. Chan, and S. G. Louie, Phys. Rev. B 34, 6656 (1986).

${ }^{26}$ G. Lehmann and M. Taut, Phys. Status Solidi B 54, 469 (1972).

${ }^{27}$ W. L. McMillan, Phys. Rev. 167, 331 (1968).

${ }^{28}$ O. Jepsen, O. K. Andersen, and A. R. Mackintosh, Phys. Rev. B 12, 3084 (1975).

${ }^{29}$ L. E. Murr, Interfacial Phenomena in Metals and Alloys (Addison-Wesley, Reading, MA, 1975), and references therein.

${ }^{30}$ D. E. Eastman Phys. Rev. B 2, 1 (1970).

${ }^{31}$ N. M. Rosengaard and H. L. Skriver, Phys. Rev. B 47, 12865 (1993).

${ }^{32}$ P. J. H. Denteneer and $W$ van Haeringen, J. Phys. C 20, L883 (1987). 
${ }^{33}$ M. Kimura, M. Mabuchi, M. Yamamoto, and T. Hashino, J. Phys. Soc. Jpn. (to be published).

${ }^{34}$ A. Zangwill, Physics at Surfaces (Cambridge University, Cambridge, 1988).

${ }^{35}$ R. Smoluchowski, Phys. Rev. 60, 661 (1941).

${ }^{36}$ K. M. Ho and K. P. Bohnen, Europhys. Lett. 4, 345 (1987).

${ }^{37}$ P. J. Feibelman, Phys. Rev. B 46, 2532 (1992).

${ }^{38}$ K. M. Ho and K. P. Bohnen, Phys. Rev. B 32, 3446 (1985).

${ }^{39}$ C. L. Fu, S. Onishi, E. Wimmer, and A. J. Freeman, Phys. Rev. Lett. 53, 675 (1984).

40 J. R. Morris (private communication).
${ }^{41}$ K. M. Ho and K. P. Bohnen, Phys. Rev. Lett. 56, 934 (1986).

42 P. J. Feibelman, D. R. Hamman, and F. J. Himpsel, Phys. Rev. B 22, 1734 (1980).

${ }^{43}$ R. L. Tapping, J. Nucl. Mater. 107, 151 (1982).

${ }^{44}$ P. J. Feibelman and F. J. Himpsel, Phys. Rev. B 21, 1394 (1980).

45 J. Hermanson, Solid State Commun. 22, 9 (1977).

${ }^{46}$ F. J. Himpsel and D. E. Eastman, Phys. Rev. B 20, 3217 (1979). 